DOI: https://doi.org/10.46630/phm.12.2020.33

Radana D. Lukajić ${ }^{1}$

Originalni naučni rad

Faculté de philologie

УДК 821.133.1.09-31 Proust M.

Université de Banja Luka

Primljen: 5. 10. 2019.

Département de langue et de littérature françaises

\title{
À LA RECHERCHE DU TEMPS PERDU : DES FANTASMES À L'ESTHÉTIQUE IDÉALISTE DANS L'CEUVRE DE PROUST
}

\begin{abstract}
Dans le travail présent, nous traitons de $\grave{A}$ la recherche du temps perdu de M. Proust, en nous appuyant sur des travaux critiques de plusieurs exégètes de l'œuvre proustienne. Une lecture transversale des études présentées dans la première section de notre travail a pour l'objectifd'illustrer que, en dépit des outils épistémologiques respectivement adoptés par les auteurs, les problématiques y traitées - la question identitaire, le rapport à l'Autre, le chronotope proustien, la fonction de la création artistique - en constituent assurément un foyer de convergence. Ensuite, nous procédons à l'extension du champ des références utilisées pour enrichir notre travail par d'autres réflexions émanant des auteurs qui traitent, explicitement ou implicitement, les problématiques majeures de $\grave{A}$ la recherche du temps perdu. Partant, les concepts philosophiques de E. Levinas nous paraissent fort pertinents pour expliciter le rapport du « je » proustien à l'Autre ; la notion de la discontinuité essentielle du temps développée par G. Bachelard nous permet de considérer la durée proustienne comme celle relevant d'une " intuition de l'instant », découverte par la mémoire affective. L'idée de l'Art rédempteur qui assure un continuum artificiel et donne un sens à la vie translatée dans l'univers esthétique fait échos à de nombreux commentaires de La Recherche.
\end{abstract}

Mots-clés : psychocritique, fantasme, chronotope proustien, mémoire, esthétique idéaliste, identité, altérite, temporalité, Art

\section{Introduction}

Se proposer de faire une analyse de l'œuvre de Proust, quelle que soit la méthode choisie, cela implique un risque certain d'être accablé par le nombre pléthorique de commentaires antérieurs. Qui plus est, les études proustiennes en tant que domaine spécialisé d'études littéraires ne sauront que décourager

${ }^{1}$ radana.lukajic@flf.unibl.org 
les tentatives des non-spécialistes dans le sens strict du terme d'offrir une interprétation nouvelle. Partant, la difficulté majeure d'ordre épistémologique oblige à une attitude $a$ priori restrictive quant au choix de littérature critique en fonction de l'approche interprétative adoptée. Une autre difficulté serait d'ordre herméneutique : vu l'étendue du champ des études proustiennes dont l'extension se poursuit, prétendre à une «nouvelle » lecture du texte ne serait-ce pas tomber dans le piège du « déjà-dit», malgré tous les scrupules déontologiques respectés ? Quelque peu acculée devant les contraintes évidentes, il nous reste une voie d'accès oblique au questionnement de l'œuvre proustienne, susceptible de faciliter notre tâche : en guise de préambule, instaurer un dialogue avec des commentaires existants pour tenter de construire une optique autre. Cela dit, pour entamer notre propos, nous tâcherons de suivre grosso modo les axes directeurs de trois études, parmi les plus notoires, susceptibles d'illustrer la variabilité des optiques choisies quant à l'interprétation de l'art proustien : La place de la madeleine de Serge Doubrovsky, L'espace proustien de Georges Poulet et Proust et les signes de Gilles Deleuze. Quelques problématiques essentielles inhérentes à l'œuvre de Proust - la question identitaire, le rapport à l'Autre, le chronotope proustien, la fonction de l'art - nous les traiterons en référence aux ouvrages critiques susmentionnés tout en amplifiant notre argumentation par les réflexions de certains auteurs qui traitent, intentionnellement ou non, les problématiques majeures de $\dot{A}$ la recherche du temps perdu. Nos propres observations sont issues de la lecture transversale des auteurs cités tout au long de cette investigation.

\section{Fantasmes, espace et signes proustiens}

Tout d'abord, attardons-nous sur l'approche psychocritique ${ }^{2}$ de $L a$ Recherche faite par Serge Doubrovsky, La place de la madeleine (2000) ${ }^{3}$. L'ouverture de l'étude en question annonce le protocole analytique de

\footnotetext{
${ }^{2}$ Selon C. Mauron, créateur de la psychocritique, une telle approche œuvre à " accroître notre intelligence des œuvres littéraires simplement en découvrant dans les textes des faits et des relations demeurés jusqu'ici inaperçus ou insuffisamment perçus, et dont la personnalité inconsciente de lécrivain serait la source " (MAURON 1983 : 13). Toujours selon Mauron, autant que cette critique se distingue de la critique classique, elle diffère considérablement de l'approche psychanalytique purement « médicale » où le but est de « diagnostiquer » l'écrivain via l’euvre réduisant celle-ci à une série de symptômes reconnus de névrose pathologique.

${ }^{3}$ Le titre complet : La place de la madeleine. Écriture et fantasme chez Proust. En effet, louvrage a pour la base une série de conférences présentées aux universités américaines et européennes entre le mois de décembre 1972 et le mois de mars 1973, alors que "le présent essai [sétant] profondément modifié en cours d'écriture » (DOUBROVSKY 2000 : quatrième de couverture). Louvrage sous sa forme définitive est publié seulement en 2000, aux éditions ELLUG de Grenoble.
} 
Doubrovsky : " Je crois qu'on a trop esthétisé, aseptisé Proust. J'aimerais lui rendre sa charge agressive, lui restituer sa violence " (DOUBROVSKY 2000 : 19). L'expérience de la madeleine, initiale et cruciale, serait, selon le critique, celle qui domine toutes les autres épiphanies proustiennes puisqu'elle seule capable de donner la clé interprétative pour la totalité de La Recherche. Essentielle, puisqu'elle nous découvre l'être dont le fantasme premier est la fusion totale avec la mère, alors que le Narrateur ${ }^{4}$ serait un être incurablement vicié par le complexe œdipien de symptomatologie classique. En se référant à certains passages de Contre Saint-Beuve $e^{5}$, Doubrovsky nous définit la situation du Narrateur (ou mieux, son arkhè-situation), le point nodal d'où vont se déployer tous les autres fantasmes du narrateur/auteur (notons que cette adéquation est un sine qua non pour ce type d'approche critique) dont l'œuvre n'est qu'une transcription esthétisée. En se référant à un texte de $\mathrm{P}$. Lejeune, le critique argue comme suit :

Forme (sexe féminin); nom (prénom de la pécheresse évangélique), cycle de la sensualité et de culpabilité, mythe de la résurrection, qui gouverne le système de La Recherche; saveur (infusion et madeleine remplaçant le pain et le vin dans un rite de « communion orale avec la mère ». [...] En somme, on pourrait dire que, grâce à la liaison masturbation/mère, par une sorte de glissement, une régression orale euphorisante (perceptible dans tout « Combray ») viendrait masquer, en la recouvrant exactement, la jubilation orgasmique, objet de jouissance interdite. Dans cette perspective, l'objet de la réminiscence se déplacerait de la madeleine à la mère, et de la mère à la masturbation. (DOUBROVSKY 2000 : 30)

Suit une analyse détaillée sur les fantasmes sexuels de Proust fixés sur la figure de la mère, alors que toutes les autres femmes aimées dont nous parle le Marcel de La Recherche ne seraient que les amours-palliatifs inefficaces pour assouvir le désir de l'union extatique et primordiale avec la génitrice. Très expéditivement, La place de la madeleine réduit le Narrateur à un "parasite » (2000 : 38, 46) qui vit sur le compte de l'Autre, alors que, paradoxalement, la relation à l'Autre est d'ordre aporétique : la présence constante de l'Autre " détermine l'absence de soi, la présence à soi exigera l'absence éternelle de l'Autre - sans lequel il est pourtant impossible d'être soi » (2000:39). Selon

\footnotetext{
${ }^{4}$ Nous reprenons ici la majuscule voulue par Doubrovsky. Tant que la référence explicite ou implicite à La place de la madeleine fait défaut, nous utiliserons « narrateur » tout au long de notre travail.

${ }^{5}$ Après l'échec d'un roman dont Proust entreprend la rédaction en 1908 et qui reste inachevé, Proust se tourne vers la réflexion théorique. La Recherche est en grande partie issue des pages de Contre Sainte-Beuve, un essai critique greffé d'un récit autofictionnel ("Récit d'une matinée »). Proust y travaille de la fin de 1908 à lété de 1909. Les pages de cet ouvrage sont restées éparpillées et malgré les efforts de plusieurs critiques, le texte n’a jamais acquis une forme achevée. Dans ce texte se trouve l'essentiel de la pensée esthétique de l'auteur qu'il va développer et parachever dans La Recherche.
} 
le critique, l'échappatoire du Narrateur - un être ankylosé dans une enfance/ adolescence obstruant sa maturation - c'est l'invention d'un langage à soi, ce qui équivaut à une invention de soi. Nous ne retiendrons pour le moment que ces quelques constats du critique. Une parenthèse nous semble ici pourtant nécessaire : le « trop esthétisé et aseptisé » quant à l'œuvre de Proust exige une réplique. La psychocritique légitime assurément une assertion du genre. Mais à force de dépister dans l'œuvre les symptômes des fantasmes a fortiori sexuels, en bon freudien qu'il se pose, Doubrovsky flirte avec la position d'un clinicien de Marcel Proust qu'il y a lieu de diagnostiquer pour nous dire toute la vérité sur les mobiles premiers (sic.) de la rédaction de La Recherche. En dehors de certains arguments pertinents, le critique se laisse emporter par moments par l'enthousiasme de « désaseptiser Proust » à tout prix pour finir dans des interprétations qui paraissent quelque peu fortuites, pour ne pas dire aberrantes : "La suppression d'autrui, nécessaire à 'l'assimilation complète d'un être', n'est pas possible tant qu'il y a 'la conversation'. [...] Écrire comme manger : c'est tuer » (2000:55); «On écrit pour se punir. On se punit pour expier» $(2000: 57)$; « [S]i la madeleine $[\ldots]$ joue comme substitution du lait, l'offre de la madeleine et du thé, c'est le lait-thé : Léthé. Fleuve de l'Oubli, c'est le bonheur du Néant » (2000: 63 ; tous les soulignements sont de l'auteur) etc. Les assertions semblables qui truffent le texte ont pour le but de désabuser le lecteur potentiel quant à la réception de La Recherche, soit pour " débarrasser cette cruelle œuvre proustienne des bondieuseries esthétisantes » (2000: 57). On a l'impression que Doubrovsky cherche à « viriliser » en quelque sorte Proust par le biais de ce recensement pédant des symptômes d'une haute frustration du sujet trahie par la " violence » et la « cruauté » qui brassent sous le poli des phrases "suralimentées $»^{6}$ de Proust. Doubrovsky - non sans une certaine palinodie épistémologique - tient cependant à affirmer explicitement que le dépistage des fantasmes libidinaux a un seul intérêt : présenter les mêmes comme les mécanismes-producteurs d'un discours littéraire (2000: 53).

Cependant, en dépit des soins du critique de cautionner son approche par l'objectif final qui serait bel et bien du ressort de la critique littéraire, le décryptage des fantasmes sexuels prévaut sur la télicité de l'analyse explicitée comme précède. Cela dit, la critique littéraire reste un peu à porte-à-faux, prête

\footnotetext{
${ }^{6}$ Selon Doubrovsky, le style "suralimenté » de Proust est révélateur du rapport existant entre l'être libidinal et lêtre créatif : "Cortège saisonnier des mets, obsession de ces 'nourritures proustiennes', bien étudiées par Jean-Pierre Richard. Dans l'univers combraisien, l'abondance, c'est la surabondance. S'alimenter, c'est se suralimenter. [...] Sitôt conçu (si l'on peut dire), le Livre donne au Narrateur, Leo Bersani l'a remarqué, les symptômes physiques de la grossesse. Il faut le 'suralimenter comme un enfant' (III, 1032). Non pas alimenter, mais suralimenter : entre son œuvre et lui, lécrivain ne peut (ne sait) que rejouer le type de rapports qui ont existé entre lui et sa mère ( (DOUBROVSKY $2000:$ 34, 48; souligné par l'auteur).
} 
à s'effriter sous la charge de complexes subliminaux recélés chez le Narrateur ${ }^{7}$. Nonobstant, le retour à l'aspect esthétique de l'œuvre était de rigueur :

La relation duelle à la mère, l'ambivalence fascinée, l'intrication l'amour-haine, l'impossibilité fondamentale d'être soi avec ou sans l'Autre : ce ne sont pas les « complexes », somme toute anecdotiques, du Narrateur, que La Recherche nous raconte, mais, à travers les complexes, accidentels, de l'écrivain, elle révèle les complexes, essentiels, de l'écriture. C'est sans doute la remarquable exemplarité proustienne que d'avoir su à ce point faire coïncider l'absolue singularité d'un destin et d'un projet, avec l'essence même de l'entreprise littéraire. Car l'écrivain moderne rejoue nécessairement avec sa langue le scénario que le Narrateur joue avec sa mère - avec l'Autre dont il est issu. (DOUBROVSKY 2000 : 131 ; souligné par l'auteur)

À l'opposé de Doubrovsky, les études critiques sur La Recherche de Georges Poulet et de Gilles Deleuze interrogent l'œuvre comme ce qui crée « une nouvelle relation axiologique à ce qui est déjà devenu réalité pour la connaissance et pour l'acte »(BAKHTINE 1978: 45 ; souligné par nous), or comme un objet esthétique sans aucune " sublimation du subliminal » programmatique comme le laisse supposer Doubrovsky. Antérieures à La place de la madeleine, il se peut qu'elles aient provoqué la réaction antiesthétique de ce dernier ${ }^{8}$.

Dans L'espace proustien (1963), Poulet pense l'œuvre de Proust comme une recherche identitaire d'un être ontologiquement perdu, perdu dans le temps mais tout aussi bien dans l'espace (POULET 1982: 19). La difficulté ressentie par le narrateur de se situer implique la notion de la non-stabilité des lieux. Au lieu de l'espace - un vide positif potentiellement rempli - il vaudrait mieux parler des « lieux » proustiens : il s'agit des îlots séparés par un espace vide, « comme une série de photographies d'une même personne ou d'un même groupe » (POULET 1982: 149). Les lieux, dans l'univers de La Recherche, comme le déduit avec génie le célèbre critique, ont besoin d'être personnalisés, et cela par le truchement de l'association des êtres et des lieux (1982 : 148). La juxtaposition des «moments retrouvés » va de pair avec la juxtaposition des sites (re)traversés. L'éclatement de l'espace, du temps, des êtres trouve le remède dans le seul moyen possible pour ce quêteur de

\footnotetext{
${ }^{7}$ Un paradoxe évident infirme l'approche de Doubrovsky : tout en citant Contre Sainte-Beuve pour y déceler les indices d'une frustration sexuelle relative au complexe œdipien, le critique exempte - délibérément ou pas - le fait que Contre Sainte-Beuve définit la pierre angulaire de la pensée esthétique de Proust : "Un livre est le produit d'un autre moi que celui que nous manifestons dans nos habitudes, dans la société, dans nos vices » (PROUST 1971 : 221).

${ }^{8}$ Notons que notre assertion n'est qu'hypothétique. Au contraire, les analyses de Philippe Lejeune, de Jean-Pierre Richard, de Leo Bersani, situées dans le même lignage critique et citées par l'auteur à plusieurs reprises, ont sûrement eu un impact considérable sur le développent argumentatif de Doubrovsky dans La place de la madeleine.
} 
l'unité ontologiquement impossible - établir les rapprochements, les liens, une chaîne d'associations par une voie métaphorique :

Poussé par un besoin profond d'unité, voulant à tout prix faire cesser l'isolement dans lequel s'enfermait, dans son univers, toute individualité originale, Marcel Proust avait inventé une méthode qui n'avait d'autre fin que d'établir partout des rapprochements. Or voici que dans l'acte même par lequel les êtres se rapprochent, ils se dédoublent, se décuplent, font apparaître une variété d'apparences, de l'une à l'autre desquelles l'esprit est sans cesse renvoyé. [...] Ne peut-on placer côte à côte, dans une proximité, qui ne serait pas une identité ? Telle est la nouvelle solution à son problème, que Proust essaie de mettre en pratique. Après la méthode de déplacement, reste celle de la juxtaposition. (POULET 1982 : 111-113 ; souligné par l'auteur).

Les lieux, comme les êtres peuvent nous quitter, mais un retour - du moins artificiel - est toujours possible. D'où le besoin de juxtaposition dont parle Poulet, puisque juxtaposer les images des lieux et des personnes qui en sont un contenu incarné, c'est procéder également à une structuration rectiligne du temps lui permettant de se présentifier. En d'autres termes, l'œuvre de Proust saurait être lue comme une lutte réitérée contre la superposition des souvenirs dans un présent actuel fort amnésique dont la tension vers le futur ensevelit les présents du passé. La circonscription linéaire du temps par une activité à la fois mnémonique et scripturaire tient de la conviction de l'auteur que le Temps est une dimension hautement spatialisée :

D'ailleurs, que nous occupions une place dans le Temps, tout le monde le sent, et cette universalité ne pouvait que me réjouir puisque c'est la vérité, la vérité soupçonnée par chacun, que je devais chercher à élucider . Non seulement tout le monde sent que nous occupons une place dans le Temps, mais cette place, le plus simple la mesure approximativement comme il mesurerait celle que nous occupons dans l'espace [...] (PROUST 1999 : 2400)

Notons que la méthodologie de Poulet n'a rien à faire avec le dépistage des symptômes/fantasmes qui relèveraient de l'instance auctoriale masquée sous un « je » diégétique : comme le remarque Jean Starobinski, la méthode de Poulet se passe de « règles » strictes, l'important étant de répondre, dans ses activités de critique, à cet appel provoqué par la fascination ressentie devant la « pensée d'autrui » (STAROBINSKI, « Préface » in POULET 1979: 11). Aucune critique docile, limitée par une épistémologie rigoureuse ne s'y opère, mais un mouvement de sympathie qui va dans le sens de l'Autre : « Derrière les formes, derrière les structures, derrière le flot continu des mots ne demeurait plus qu'une seule chose : une pensée sans forme, toujours différente d'ellemême dans ses manifestations successives mais toujours inaltérablement ellemême dans son fond » (POULET 1971 : 202). 
Dans Proust et les signes (1964), Deleuze part de la thèse d'une esthétique idéaliste de Proust: À la recherche du temps perdu jalonne, selon Deleuze, l'itinéraire d'une infatigable quête de la vérité qui réside dans les essences, mais en dehors du cadre classique du logos grec. Qualifier La Recherche comme un roman d'apprentissage, entre autres, est tout à fait justifié si tant est que l'apprentissage signifie la propédeutique du décryptage des signes : « Tout ce qui nous apprend quelque chose émet des signes, tout acte d'apprendre est une interprétation de signes ou de hiéroglyphes. L'œuvre de Proust est fondée, non sur l'exposition de la mémoire, mais sur l'apprentissage des signes » (DELEUZE 2007 : 11).

L'héritage platonicien chez Proust y est mis en évidence avec une méthode rigoureusement élaborée. Selon le critique, les signes restent sans importance sans qu'il n'y ait une intelligence qui y découvre les essences cachées que seull'Art saurait rendre ipsofacto signifiantes. Penser les essences, c'est penser la pluralité de leurs émanations aussi bien que la pluralité des essences elles-mêmes. Cependant, une différence patente intervient dans la pensée proustienne par rapport au modèle platonicien - tant que chez Platon l'Idée, une Essence stable, est toujours en avant, le point d'arrivée d'une réminiscence, chez Proust « la fusion mutuelle », " l'instable opposition » sont tributaires d'un état d'âme, et non d'un état de choses ou de monde :

L'essence selon Proust [...] n'est pas quelque chose de vu, mais une sorte de point de vue supérieur. [...] Mais l'important est que le point de vue dépasse l'individu, non moins que l'essence, l'état d'âme : le point de vue reste supérieur à celui qui s'y place, ou garantit l'identité de tous ceux qui y atteignent. Il n'est pas individuel, mais au contraire principe d'individuation. C'est là précisément l'originalité de la réminiscence proustienne : elle va d'un état d'âme, et de ses chaînes associatives, à un point de vue créateur ou transcendant - et non plus, à la manière de Platon, d'un état du monde à des objectivités vues. (DELEUZE 2007 : 133-134)

Pour la pensée proustienne, l'essence, c'est en premier lieu la différence (DELEUZE 2007 : 53). Le trouble ressenti par différenciation et parcellisation tous azimuts est surmonté par la perception des signes qui, l'intelligence l'aidant, attribue à l'anamnèse la qualité purement créative : créer c'est se ressouvenir, mais l'inverse vaut également - se ressouvenir, c'est créer. Une seule issue est appréhendée, après le long circuit d'apprentissage de signes : la structure formelle d'une œuvre d'art est seule capable d'assurer une unité, une unité des essences conçues comme des points de vue supérieurs (DELEUZE 2007 : 119).

Si esthétisation de Proust il y a, elle est sûrement à l'œuvre dans l'approche analytique de Deleuze. Cependant, malgré les divergences entre 
les trois études ${ }^{9}$ dont nous avons essayé de suivre les linéaments majeurs, une lecture synoptique nous permet d'en dégager un foyer de convergence fédérant les problématiques suivantes que l'œuvre déploie : le rapport à l'Autre, y compris le Temps, étroitement lié à la problématique identitaire et la conception de l'art comme producteur d'une réalité transcendante.

\section{La question identitaire - de la fragmentation universelle à l'unité artificielle}

Le rapport aporétique à l'Autre décrit par Doubrovsky - « la présence continuelle détermine l'absence à soi, la présence à soi exigera l'absence éternelle de l'Autre » $(2000: 39)$ - ne peut donc pas se résoudre sans une autogenèse, un « accouchement de soi » dans un langage neuf à partir duquel s'érige l'œuvre. Mais, par une logique suprême, ce moi ectoplasmique dit d'une manière encore plus virulente un manque de soi (DOUBROVSKY 2000 : 122). Les apories en apparence insolubles pour l'instance narrant sont productrices de l'écriture qui est un artefact non seulement de l'unité, mais aussi de l'identité : «Je naît du Je, qui est le même que l'autre» (2000 : 109 ; souligné par l'auteur). La prétendue autofécondation débouche, comme le démontre Doubrovsky, non sur le constat de l'altérité irrémédiable de soi à soi, mais sur le refus de toute identité qui serait repérable au niveau du langage : nous avons affaire à un « je » non identifiable par un nom, puisque nommer c'est déjà aliéner (2000 : 100-101). Comment alors expliquer l'obsession du narrateur par les noms propres - noms de familles, nom de paysages auxquels l'auteur consacre une partie considérable de La Recherche? À l'instar de son œuvre qui nomme l'innommable - les noms, à plus forte raison les noms de famille ont la fonction extensive - l'exister d'un être se greffe sur celui d'un paysage dans un brassage temporel vertigineux. À titre d'exemple, nous citons un parmi de nombreux passages qui ponctuent le soliloque narratif :

Les noms cités avaient pour effet de désincarner les invités de la duchesse, lesquels avaient beau s'appeler le prince d'Argigente ou de Cystria, que leur masque de chair et d'inintelligence ou d'intelligence communes avait changés en hommes quelconques, si bien qu'en somme j'avais atterri au paillasson du

\footnotetext{
${ }^{9}$ Quant aux trois études présentées, la ligne de clivage sépare plutôt l'approche de Doubrovsky d'un côté, et celles de Poulet et de Deleuze de l'autre dont l'objet est une pensée consciente qui structure un discours idéologique et esthétique et non la traduction des mécanismes subconscients du narrateur/auteur en un discours esthétique. À ce propos, une contradiction dans les termes évidente est à relever dans La place de la madeleine : l'argumentation de Doubrovsky laisse croire que le subliminal n'est point sub-liminal, puisqu'une activité bien consciente est en œuvre dans la soi-disant transformation des fantasmes en un discours littéraire (DOUBROVSKY 2000 : 53).
} 
vestibule, non pas comme au seuil, ainsi que je l'avais cru, mais au terme du monde enchanté des noms. Le prince d'Argigente, lui-même, dès que j'eus entendu que sa mère était de Damas, petite-fille du duc de Modène, fut délivré, comme d'un compagnon chimique instable, de la figure et des paroles qui empêchaient de le reconnaître, et alla former avec Damas et Modène, qui eux n'étaient que des titres, une combinaison infiniment plus séduisante. (PROUST 1999 : 1161-1162)

Cette combinaison crée de toute évidence non seulement une nouvelle entité « être-lieu », mais opère de surcroît une dilatation de l'être et de l'espace $^{10}$. L'extension ainsi opérée a pour l'objectif de dégager une essence de cette symbiose tout en produisant une réduction des distances : le « je » proustien est sans aucun doute porté par un désir de rapprochement, d'abolition de tout intervalle par ce procédé de distension associative, tout comme par celui de juxtaposition dont parle Poulet. Le fractionnement universel, la distance omniprésente, l'isolement de tout ce qui phénoménologiquement est sont les seules évidences auxquelles le « je » de La Recherche veut remédier en agençant une trame narrative qui restitue, pièce par pièce, les fragments de l'univers dont le morcellement contamine tout - les lieux, le temps, les êtres, les choses. On dirait que, entre autres, La Recherche nous relate l'impossibilité d'une communication à part entière à laquelle l'être tend (ce qui n'est pas loin d'un désir de communion), puisque le monadisme est ubique et irréversible. Dès lors, un seul expédient s'impose : créer une unité factice à partir de l'éclatement qui se retrouve à tous les niveaux de présences phénoménologiques. L'éclectisme conscient, résultant du cycle des célèbres extases du narrateur, tend à pérenniser, par le biais d'une unité factice, les débris éparpillés d'une existence aléatoire.

Malgré cette tentative de réduction, voire d'abolition, de la distance par des procédés narratifs, l'être proustien semble être condamné à un solipsisme ontologique, puisque chaque nouvelle expérience radicalise le rapport de distance entre tout ce qui existe, une distance insurmontable, puisque chaque phénomène, à plus forte raison chaque être, reste un vase clos que le désir de Marcel, transformé en écriture, rende contigu à un autre vase clos : «En vérité, il existe une activité, un pur interpréter, pur choisir, qui n'a pas plus de sujet que d'objet, puisqu'elle ne choisit moins l'interprète que la chose à interpréter, le signe et le moi qui le déchiffre » (DELEUZE 2007 : 154-155 ; souligné par l'auteur).

Notons que l'interprétation et la sélectivité dont parle Deleuze ne sont plus, comme dans toutes les fictions ou les auto-graphies, les activités d'une instance ontologiquement stable. Le moi proustien est un « je » lymphatique, « choisi » au gré des circonstances, dans un mouvement constant de

${ }^{10}$ En ce qui concerne l'importance des noms propres dans À la recherche du temps perdu, $c f$. notamment Barthes (1972 : 118-130). 
transgression de limites temporelles et spatiales : le Marcel de La Recherche n'est qu'un grand absent, un inconnu, un morphème creux qui nous leurre par des références aux lieux, temps, milieux, prénoms. Une vision globale d'un « moi » ne prétend aucunement à une quelconque recomposition de moi à partir des données crues : au contraire, les données crues ne signifient plus rien, puisque la vie - ressouvenue dans un souvenir réitéré - est, somme toute, un grand pan du temps perdu dans la matrice du Grand Temps. L'irrémédiable défaut de soi à soi n'est cependant pas un échec issu de la tentative de l'émancipation par rapport à l'Autre dont l'Imago archétypale serait la Mère, comme le laisse conclure Doubrovsky. La problématique relation de soi à soi se révèle par à coup, à travers les expériences successives de non-coïncidences. Cela dit, toutes les premières pages de La Recherche peuvent être lues comme une dramatique confrontation à une seule évidence : l'effritement, l'instabilité, le vacillement qui régissent toutes les données quantitatives et qualitatives de l'existence :

Toujours est-il que, quand je me réveillais ainsi, mon esprit s'agitant pour chercher, sans y réussir, à savoir où j'étais, tout tournait autour de moi dans l'obscurité, les choses, les pays, les années. Mon corps, trop engourdi pour remuer, cherchait, d'après la forme de sa fatigue, à repérer la position de ses membres pour induire la direction du mur, la place des meubles, pour reconstruire et pour nommer la demeure où il se trouvait. Sa mémoire, la mémoire de ses côtes, de ses genoux, de ses épaules, lui présentait successivement plusieurs chambres où il avait dormi, tandis qu'autour de lui les murs invisibles, changeant de place selon la forme de la pièce imaginée, tourbillonnaient dans les ténèbres. (PROUST 1999: 15)

L'obscurité, l'impossibilité de se situer, une mémoire faillible, la scission d'avec le corps : les vocables-clés qui illustrent toute la situation ontologique de l'être proustien. Retenons que l'étrangeté, la distance qui s'établit de soi à soi sont accentuées aussi sur le niveau sémantique : le corps devient une entité « pour-soi », un « il » fragmenté en parties autonomes, avec «sa » mémoire à lui. Le sujet s'objective, tandis qu'un creux fondamental s'ouvre à partir duquel va se déployer tout le reste de l'auto-narration.

La relation à l'Autre répète le même échec de communion par le fait même que le rapport à l'Autre est celui de deux monades qui demeurent irrémissiblement non-appropriables. Dans Le temps et l'autre (1979), E. Levinas définit l'être comme irrémissiblement clôturé dans sa solitude puisque c'est le seul contenu dans lequel peut se réaliser son exister : " On peut tout échanger entre êtres saufl'exister. Dans ce sens, être, c'est s'isoler par l'exister. C'est par l'exister que je suis sans portes ni fenêtres, et non par un contenu quelconque qui serait en moi incommunicable» (LEVINAS 1983 : 21). Selon Levinas, l'altérité inhérente à l'être transmuée dans une condition a priori 
positive, c'est l'altérité des sexes. La dualité sexuelle conçue comme un tout dans la relation amoureuse inciterait à la concevoir comme une fusion, mais c'est justement le contraire qui s'opère dans la relation entre les deux sexes : « Le pathétique de l'amour consiste dans une dualité insurmontable des êtres. C'est une relation avec ce qui se dérobe à jamais » (1983: 78).

En traitant de ce type de relation dans l'œuvre de Proust, Levinas allègue comme suit :

Marcel n'aima pas Albertine, si l'amour est une fusion avec autrui, extase d'un être devant les perfections de l'autre ou la paix de la possession. Demain il rompra avec la jeune femme qui l'ennuie. Il fera ce voyage depuis longtemps projeté. Le récit de l'amour de Marcel est doublé d'aveux destinés semble-t-il à mettre en question la consistance même de cet amour. Mais ce non-amour est précisément l'amour, la lutte avec l'insaisissable, - la possession, cette absence d'Albertine -, sa présence. (LEVINAS 1976 : 155)

Or l'échec capital du « je » proustien est à chercher dans sa tentative de s'approprier l'exister de l'Autre, même au-delà de sa mort, ce dont il se rend compte par un lent processus d'apprentissage. Le mystère de l'Autre demeure inaliénable. Le paroxysme de l'étrangeté foncière de l'Autre réside dans le fait que la mort rend cette étrangeté encore plus radicale : Albertine continue d'émaner les signes de mystère de son exister même après sa mort ; les découvertes que le narrateur fait au sujet de la disparue représentent le climax de l'impossibilité d'atteindre la vérité sur l'Autre. La vérité n'est l'affaire du factuel, mais de l'extraction des essences qui constituent une vérité pour soi, une fois qu'elles sont translatées dans l'univers de l'Art. L'œuvre esthétique est un système autoréférentiel, autosuffisant, produisant sur elle-même ses propres effets (DELEUZE 2007 : 185).

L'impossible fusion avec l'Autre - la relation amoureuse en figure une

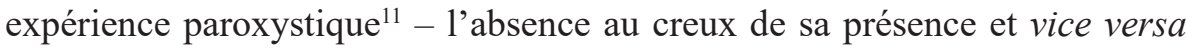
constituent les apories de la relation à l'Autre qui ne peut pas échapper à la condition d'un exister solitaire et immanent. La seule solution pour un sujet qui se sent vide de sa propre essence - une expérience poignante bâtie sur une série de désabusements - et par là, de son propre exister, c'est inventer

\footnotetext{
${ }^{11}$ À ce sujet, notons que Deleuze insiste sur la relation amoureuse fils-mère, mais à la différence de Doubrovsky, l'amour pour la mère n'est pas le fantasme érotico-amoureux paradigmatique pour toutes les autres expériences d'amour du narrateur : " [L]image de la mère n'est peut-être pas le thème le plus profond, ni la raison de la série amoureuse : il est vrai que nos amours répètent nos sentiments pour la mère, mais ceux-ci répètent déjà d’autres amours [l'amour de Swann pour Odette], que nous n'avons pas nous-mêmes vécues (sic.). La mère apparaît plutôt comme la transition d'une expérience à une autre, la manière dont notre expérience commence, mais déjà s'enchaîne avec d'autres expériences qui furent faites par autrui. À la limite, l'expérience amoureuse est celle de l'humanité tout entière, que traverse le courant d'une hérédité transcendante » (DELEUZE 2007 : 89).
} 
un nouveau rapport entre la vie et l'Art : l'Art seul peut transcender une vie sans signification, vaine, toute forgée de banalités. L'Art est non seulement l'espace où sont décantées, par une activité esthétique, les essences, mais elle est également productrice à la fois de l'identité et de la vie - grâce aux instants retrouvés dans la plénitude de leurs essences présentifiées, le vécu a pu être recollé dans un objet esthétique à l'armature solide : A la recherche du temps perdu. $^{12}$

\section{Proust et l'intuition de l'instant}

Quant à la perception du temps chez Proust, à l'opposé des rapprochements qu'on a souvent effectués entre la durée bergsonienne et la prétendue durée proustienne, on soutiendra la thèse que le génie de Proust a bien compris que la durée temporelle n'est pas une donnée immédiate de la conscience - au contraire, elle est produite : la seule réalité du Temps est celle de sa discontinuité essentielle. Gaston Bachelard en traite longuement dans son essai L'intuition de l'instant (1931) ${ }^{13}$, en s'appuyant aux réflexions anti-bergsoniennes de Gaston Roupnel. Les points de convergence entre l'herméneutique bachelardienne et la perception du temps proustienne sont nombreux, ce que nous essaierons d'illustrer dans les lignes qui suivent.

À l'issue de notre parcours effectué jusqu'à présent, un constat s'impose : la non-continuité, la dispersion, la multiplication affectent le temps, l'espace, les êtres. Si La Recherche annonce dès son titre le thème majeur - la recherche du temps perdu, perdu dans le double sens d'un temps dilapidé et d'un temps englouti dans un passé chronologique, c'est que tout «l'apprentissage » du narrateur, bâti sur l'histoire des déceptions et frustrations multiples, tient d'un devenir qui est par son essence temporel. La matrice du Grand Temps - insondable, inconcevable - absorbe le temps du vécu et se pose comme un Autre ultime puisqu'il est impossible d'empiéter sur son devenir propre qui se situe dans la dialectique de l'écoulement, d'un à-venir, alors que «[1]'avenir, c'est l'autre » (LEVINAS 1979 : 64). Cependant, les illuminations du Marcel le narrateur semblent suspendre le devenir du Temps et découvrir

\footnotetext{
${ }^{12}$ "Signifiant et signifié fusionnent par un court-circuit poétiquement nécessaire, mais ontologiquement gratuit et imprévu. Le langage chiffré ne se réfère pas à un cosmos objectif, extérieur à l'œuvre ; sa compréhension n'a de valeur qu'à l'intérieur de l'œuvre et se trouve conditionnée par la structure de celle-ci. L’ouvre en tant que Tout propose de nouvelles conditions linguistiques auxquelles elle se soumet et devient elle-même la clef de son propre chiffre » (U. Eco, L'ouvre ouverte, Paris : Seuil, p. 231 ; cité in DELEUZE 2007 : 187-188).

${ }^{13}$ L’ouvre développe la thèse de la discontinuité du temps de G. Roupnel (1971-1946), historien, philosophe et écrivain français. Cette thèse est exposée, entre autres, dans le traité philosophique Siloë (1927) de ce dernier qui sert de base aux réflexions bachelardiennes.
} 
toute la plénitude des présents du passé. Selon Bachelard, le seul moyen d'appréhender le Temps, c'est d'éliminer l'idée d'une durée quelconque : le Temps est fondé sur les instants, donc sur une discontinuité absolue. La vie, c'est la discontinuité des actes (et non une filature des actions), qui sont toujours des décisions instantanées. L'instant doit être compris comme un « point de l'espace-temps » (BACHELARD 1992 : 31). Qu'est ce qui nous fait alors croire à une durée effective, continue ? C'est l'habitude, " comme une assimilation routinière de la nouveauté » (BACHELARD 1992 : 64). À Bachelard de déduire dans les termes suivants :

L'identité globale est faite alors de redites plus ou moins exactes, de reflets plus ou moins détaillés. Sans doute l'individu s'efforce de copier aujourd'hui sur hier ; cette copie est d'ailleurs aidée par la dynamique des rythmes, mais ces rythmes ne sont pas tous au même point de leur évolution et c'est ainsi que la plus solide des permanences spirituelles, d'identité voulue, affirmée dans un caractère, se dégrade en ressemblance. [...] C'est un faisceau d'habitudes qui nous permet de continuer d'être dans la multiplicité de nos attributs en nous laissant l'impression que nous avons été, alors même que nous ne pourrions trouver en nous, comme racine substantielle, que la réalité que nous livre l'instant présent. (BACHELARD 1992 : 70-71, 74).

On sait très bien quelle importance joue l'habitude dans la vie du narrateur - le Marcel de La Recherche est un être de l'habitude par excellence. Le temps de La Recherche est celui de la réitération, d'une série d'habitudes qui accentuent la stagnation intérieure : la redécouverte des moments perdus par la magie mnémonique opérée par la voie sensationnelle fait l'irruption dans cette durée artificielle créée par la répétition. La résurrection d'un moment du passé découvre, telle une hiérophanie, toute la richesse du temps en instants qui sont, notons bien, non seulement revécus, mais rendus : le souvenir qui surgit d'une tasse de thé, d'une serviette rigide, d'un pavé irrégulier, de l'écoute d'une sonate etc. donne accès à une réalité radicalement nouvelle puisque saturée d'instants dont l'intuition, au temps de l'expérience originelle, faisait défaut. Et à Proust d'instaurer un nouveau concept de la réalité :

Une image offerte par la vie nous apportait en réalité à ce moment-là des sensations multiples et différentes. La vue, par exemple, de la couverture d'un livre déjà lu a tissé dans les caractères de son titre les rayons de lune d'une lointaine nuit d'été. Le goût du café au lait matinal nous apporte cette vague espérance d'un beau temps qui jadis si souvent, pendant que nous le buvions dans un bol de porcelaine blanche, crémeuse et plissée qui semblait du lait durci, quand la journée était encore intacte et pleine, se mit à nous sourire dans la claire incertitude du petit jour. Une heure n'est pas qu'une heure, c'est un vase rempli de parfums, de sons, de projets et de climats. Ce que nous appelons la réalité est un certain rapport entre ces sensations et ces souvenirs qui nous entourent simultanément [...] rapport unique que l'écrivain doit retrouver pour 
en enchaîner à jamais dans sa phrase les deux termes différents. (PROUST 1999 : 2279-2280)

L'heure proustienne n'est pas seulement pleine, elle est complète, puisque c'est «l'heure où tous les instants vivants seraient sentis, aimés, pensés » (BACHELARD 1992 : 48). Poulet déjà a bien compris que la durée proustienne se situe à rebours de celle de Bergson dont Proust aurait subi une influence. Il est bien évident que la durée en soi est un concept auquel Proust oppose une durée toute construite (« enchaîner à jamais »). La découverte de l'instant plein, réalisée par la mémoire affective, est à la base de la construction d'une identité dans l'espace-temps de l'œuvre d'art - un objet isolé qui répète sa solitude remplie d'instants riches en essences qui se réactualisent dans les lectures renouvelées.

\section{Conclusion}

Dans le travail présent, nous sommes partie d'une mise en regard de trois études, parmi les plus notoires, traitant de l'œuvre de Proust et dont chacune propose une lecture différente de La Recherche. Ce faisant, nous nous sommes permis d'émettre certains commentaires concernant les ouvrages présentés. Ainsi la psychocritique de Doubrovsky nous a-t-elle paru en outrance " clinique ", toute portée à soutenir la thèse de départ ellemême quelque peu présomptueuse : une sur-esthétisation généralisée de l'œuvre de Proust qu'il aurait fallu par conséquent désesthétiser pour lui rendre sa charge « de violence » et « de cruauté ». Les études respectives de Poulet et Deleuze analysent $\dot{A}$ la recherche du temps perdu comme un objet a priori esthétique et non comme un vécu aux frustrations esthétisées; les deux ouvrages sont axés, chacun obéissant à des prémisses herméneutiques différentes et cependant concomitantes, sur l'interrogation des dispositifs à la fois idéologiques et esthétiques de La Recherche. Insistons cependant sur le fait suivant : la lecture transversale des études présentées dans la première section de notre travail a eu pour l'objectif ultime d'illustrer que, en dépit des différences épistémologiques et herméneutiques entre les études présentées, les problématiques y traitées - la question identitaire, le rapport à l'Autre, le chronotope proustien, la fonction de la création artistique - en constituent une aire de convergence indéniable.

Dans les développements ultérieurs de notre travail, nous avons essayé, toujours en nous appuyant sur les analyses présentées, d'amplifier notre argumentation par des réflexions d'autres auteurs aussi bien que de proposer quelques nouvelles pistes de lecture de La Recherche. La fragmentation tous azimuts, la multiplication, la non-continuité, la structure kaléidoscopique 
de l'univers auxquelles fait face le « je » de la narration affectent presque toutes les notions-clés interrogées par l'œuvre de Proust : l'identité devient « non-identifiable », le rapport à l'Autre s'avère être aporétique, la temporalité se meut en une dimension hautement spatialisée. L'Art apparaît comme un nouveau rapport à la réalité, apte à condenser le morcellement et les distances ubiques pour créer un continuum, certes artificiel, mais cependant salvateur. Les rapprochements que nous avons faits entre Proust et Bachelard quant à la conception du temps ont eu pour l'objectif de rejeter l'idée de la durée bergsonienne qui aurait été empruntée par Proust à la philosophie de ce dernier et de montrer que la mémoire affective a affaire à l'instant comme la seule mesure du temps. La discontinuité du temps est à l'origine de la découverte de la plénitude d'un Temps saccadé, extrêmement riche en instants fédérateurs d'impressions multiples.

L'histoire de la conquête d'une vocation, la monumentale cathédrale littéraire de Proust? Oui, mais seulement dans la mesure où cette vocation peut assurer un salut, une cohésion quelque postiche qu'elle soit. L'idée de l'Art rédempteur est incontestablement une notion-phare dans l'esthétique proustienne : 1'Art assure non seulement une nouvelle relation entre les contingences d'une vie en apparence banale et leur transcription esthétique, mais aussi entre la condition sociale - et humaine - et la condition artistique. L'Art est une vie authentique, sans commune mesure avec la vie de tous les jours. Certes, la vie ainsi transcendée n'assure qu'une immortalité précaire - « [1]a durée éternelle n'est pas plus promise aux œuvres qu'aux hommes » (PROUST 1999 : 2398) - mais la vie translatée en une forme esthétique se pourvoie d'un sens et, enfin, d'une essence. Tel est le vrai sujet de $\grave{A}$ la recherche du temps perdu - la recherche d'une esthétique idéaliste qui sauve l'essence des êtres et des phénomènes multiples et assure, enfin, la survie non à un moi social mais à un moi créateur. Si agressivité et cruauté il y a dans l'œuvre de Proust, elles ne sont pas dues à un diapason restreint de fantasmes érotiques, mais à cette lutte acharnée et déchirante pour transformer le désir d'être et d'avoir en désir de (re)créer :

La vraie vie, la vie enfin découverte, la seule vie par conséquent pleinement vécue, c'est la littérature. [...] Ce travail qu'avaient fait notre amour-propre, notre passion, notre esprit d'imitation, notre intelligence abstraite, nos habitudes, c'est ce travail que l'art défera, c'est la marche en sens contraire, le retour aux profondeurs où ce qui a existé réellement gît inconnu de nous, qu'il nous fera suivre. (PROUST 1999 : 2285) 


\section{Références bibliographiques}

BACHELARD 1992 : BACHELARD, Gaston. L'Intuition de l'instant. Paris : Stock, 1992.

BAKHTINE 1978 : BAKHTINE, Mikhaill. Esthétique et théorie du roman. Paris : Gallimard, 1978.

BARTHES 1972 : BARTHES, Roland. Le degré zéro de l'écriture. Paris : Seuil, 1972.

DELEUZE 2007 : DELEUZE, Gilles. Proust et les signes. Paris : PUF, 2007.

DOUBROVSKY 2000 : DOUBROVSKY, Serge. La place de la madeleine. Écriture et fantasme chez Proust. Grenoble : ELLUG, 2000.

LEVINAS 1983 : LEVINAS, Emmanuel. Le temps et l'autre. Paris : PUF, 1983.

LEVINAS 1976 : LEVINAS, Emmanuel. Noms propres. Saint-Clément-deRivière : Fata Morgana, 1976.

MAURON 1983 : MAURON, Charles. Des métaphores obsédantes au mythe personnel. Paris : Joseph Corti, 1983.

POULET 1982 : POULET, Georges. L'espace proustien. Paris : Gallimard, 1982.

POULET 1979 : POULET, Georges. Les métamorphoses du cercle. Préface de Jean Starobinski. Paris : Flammarion, 1979.

POULET 1971 : POULET, Georges. La Conscience critique. Paris : Joseph Corti, 1971.

PROUST 1971 : PROUST, Marcel. Contre Sainte-Beuve. (Précédé de Pastiches et mélanges. Et suivi de Essais et articles). Paris : Gallimard, « Pléiade », 1971.

\section{Source}

PROUST 1999 : PROUST, Marcel. À la recherche du temps perdu. Paris : Gallimard, éd. Quarto, 1999.

\section{Radana Lukajić}

\section{U POTRAZI ZA IZGUBLJENIM VREMENOM: OD FANTAZAMA DO IDEALISTIČKE ESTETIKE U PRUSTOVOM DJELU}

U predstavljenom radu, bavili smo se analizom Prustovog monumentalnog opusa $U$ potrazi za izgubljenim vremenom oslanjajući se na kritičke studije nekolicine egzegeta Prustovog djela. S tim u vezi, kako bismo istaknuli varija- 
cije $\mathrm{u}$ interpretativnim pristupima djelu, $\mathrm{u}$ prvom odjeljku rada predstavili smo, u osnovnim crtama te sa izvjesnim metakritičkim osvrtom, tri studije koje polaze od različitih epistemoloških i hermeneutičkih premisa: Mjesto madlene Serža Dubrovskog, Prustovski prostor Žorža Pulea te Prust i znakovi Žila Deleza. Ukazali smo na nemale razlike po pitanju tumačenja Prustovog djela, nadasve između psihokritičke studije Serža Dubrovskog s jedne strane, i studija potonja dva autora koja Potrazi prilaze kao estetsko-ideološkoj cjelini koja svjesno stvara novi aksiološki odnos sa realnošću, a ne kao estetizaciji subliminalnih fantazama kako se da iščitati iz Mjesta madlene. No, uprkos istaknutim razlikama, sve tri studije se dotiču, manje ili više, ključnih problematika Prustovog djela: identitet, prustovski hronotop, odnos prema Drugom, te funkcija umjetničkog djela. U daljim razradama pozabavili smo se ovim problematikama proširujući dijapazon citiranih autora: s tim u vezi, filozofski koncepti E. Levinasa poslužili su nam za pojašnjenje Prustovog pojma identiteta i nadasve odnosa ja-Drugo; koncepcija vremena kao krajnje diskontinuirane dimenzije koja je zasnovana na trenutku a ne na trajanju G. Bašlara, činila nam se u potpunosti saobraznom sa Prustovim poimanjem vremena. Time smo opovrgnuli uveliko prihvaćenu ideju o uticaju Bergsonove filozofije na Prustovu koncepciju vremena. Shvatanje umjetnosti kao ,istinskog života“ sa obesmrćenim esencijama u uskoj je vezi sa „intuicijom trenutka“ G. Bašlara, kao i argumentima koje obrazlažu u prethodno navedenim studijama Pule i Delez.

Ključne riječi: psihokritika, fantazam, prustovski hronotop, sjećanje, idealistička estetika, identitet, alteritet, temporalnost, Umjetnost 